\title{
„Es war kein Job, es war eine Berufung“ - Gouvernementale Führung, fragmentierte Subjekte und contre-conduite in der nichtstaatlichen Entwicklungspraxis
}

\author{
Kirsten Linnemann \\ Institut für Geographie, Westfälische Wilhelms-Universität Münster, Germany \\ Correspondence: Kirsten Linnemann (k.linnemann@uni-muenster.de)
}

Received: 2 July 2018 - Revised: 12 April 2019 - Accepted: 2 May 2019 - Published: 27 May 2019

\begin{abstract}
Kurzfassung. With their donation appeals aid organisations procure a polarised worldview of the self and other into our everyday lives and feed on discourses of "development" and "neediness". This study investigates how the discourse of "development" is embedded in the subjectivities of "development" professionals. By approaching the topic from a governmentality perspective, the paper illustrates how "development" is (re-)produced through internalised Western values and powerful mechanisms of self-conduct. Meanwhile, this form of self-conduct, which is related to a "good cause", also gives rise to doubts regarding the work, as well as fragmentations and shifts of identity. On the one hand, the paper outlines various coping strategies used by development professionals to maintain a coherent narrative about the self. On the other hand, it also shows how doubts and fragmentations of identity can generate a critical distance to "development" practice, providing a space for resistant and transformative practice in the sense of Foucauldian counter-conduct.
\end{abstract}

\section{Einleitung}

Der Imperativ der „Entwicklung“ ist mit seinen essentialistischen Weltbildern von ,entwickelt“ und ,unterentwickelt“ und seinen kolonialen Wurzeln bis heute fest in gesellschaftliche Strukturen eingeschrieben. Als Eckpfeiler der gegenwärtigen globalen Ordnung begegnet uns der Entwicklungsdiskurs mehr oder weniger subtil in Politik, Medien, Schule und anderen gesellschaftlichen Institutionen.

In diesem Zusammenhang nimmt auch das Feld der sogenannten „Entwicklungszusammenarbeit“ (,EZ“) eine prominente Rolle ein. Spendenaufrufe von Hilfsorganisationen im öffentlichen Raum, TV-Spendengalas in der Vorweihnachtszeit und Briefwerbung für „Kinderpatenschaften“ spielen häufig mit dichotomen Repräsentationsmustern von Not, Elend und „Unterentwicklung“ auf der einen und aktiven, fähigen und selbstlosen „Helfer*innen“ auf der anderen Seite (vgl. z.B. Bendix, 2018; Eriksson Baaz, 2005; Kiesel und Bendix, 2010; Linnemann und Reuber, 2015; Strüver, 2007).
Gleichzeitig üben entwicklungskritische Strömungen wie Post-Development und Postkolonialismus (z.B. Ashcroft et al., 2006; Escobar, 1995; Kapoor, 2008; McEwan, 2009) ${ }^{1}$ sowie dekoloniale Theorien (z.B. Mignolo, 2012 [2006]; Mignolo und Walsh, 2018; Quijano, 2000) seit vielen Jahren Kritik an den konzeptionellen Fallstricken und politischen Implikationen des Entwicklungsdiskurses, an eurozentrischen Wissens- und Repräsentationssystemen, essentialisierten Weltbildern und fortdauernden kolonialen Machtasymmetrien. Diese macht- und entwicklungskritischen Bewegungen werden von Stimmen aus der Entwicklungspraxis flankiert, die die Auswüchse, die Erfolge und den Sinn der „EZ“ auf der Grundlage eigener Erfahrungen kontrovers diskutieren (z.B. Polman, 2010).

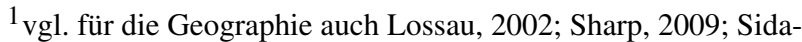
way, 2007 und die Beiträge des Themenheftes „Post-Development“ in der Geographica Helvetica, 67 (3) (einleitend: Neuburger und Schmitt, 2012).
} 
Vor diesem gesellschaftlichen Hintergrund stellt sich die Frage, wie die zahlreichen Mitarbeiter*innen in „EZ“Organisationen die gegenläufigen Tendenzen von Entwicklungsdiskurs und seiner Kritik verhandeln: Wie schreiben sich die tradierte Großerzählung der „Entwicklung“ und ihr moralischer Imperativ, „Gutes“ zu tun, in die Identitäten von Entwicklungspraktiker*innen ein? Welche Rolle spielen die alltäglichen Praxiserfahrungen und Erlebnisse im Job? Wie differenziert, kritisch und selbstreflexiv setzen sie sich mit ihrer Arbeit, hegemonialen Weltbildern und globalen Abhängigkeitsverhältnissen auseinander? Im Mittelpunkt dieses gouvernementalitätstheoretisch informierten Beitrages steht somit die wechselseitige Beziehung zwischen wirkmächtigen Weltbildern und Subjektivierungsprozessen von Entwicklungspraktiker*innen. Die empirische Grundlage bilden narrativ-biographische Interviews mit Menschen aus dem Kontext der nichtstaatlichen „Entwicklungszusammenarbeit“ und „Humanitären Hilfe“, die mit aussagenanalytischen Mikromethoden der Diskursforschung (Angermüller, 2007; Glasze, 2007; Mattissek, 2008, 2009) ausgewertet wurden.

Mit dieser Ausrichtung schließt der Beitrag an eine Reihe von Arbeiten an, die sich Fragen der ,Entwicklung“ mehr oder weniger konsequent aus einer gouvernementalitätstheoretischen Perspektive nähern (vgl. z.B. Bendix, 2018; Hart, 2004; Li, 2007; die Beiträge in Mezzadra et al., 2013; Rossi, 2004; Ziai, 2016). So analysiert z.B. Daniel Bendix (2018) deutsche Entwicklungspolitik und -praxis u.a. anhand von Spendenwerbung und Bildungsarbeit in Deutschland aus einer postkolonialen und diskurstheoretisch informierten Perspektive. Das Gros der Foucault-inspirierten Arbeiten in diesem Feld richtet seinen Fokus jedoch auf die Zielregionen und Empfänger*innen der „Hilfe“ (vgl. z.B. Bryant, 2002; McDonald, 1999) und/oder auf neoliberale Formen der Gouvernementalität (Ferguson und Gupta, 2002). Durch die Konzentration auf die Subjektivierungsprozesse von Entwicklungspraktiker*innen ergänzt dieser Beitrag die bisherigen Forschungen somit um eine neue Perspektive.

Gleichzeitig knüpft er damit an eine Forschungsrichtung der anglophonen Kulturanthropologie an, die eine ähnliche Kritik am üblichen Fokus der Entwicklungsforschung formuliert und sich mit Fragen der Identität, ebenso wie mit den Spannungen und Herausforderungen in der alltäglichen Arbeit von „development professionals“ und ,aid workers“ (Harrison, 2013: 263, 265) beschäftigt (vgl. z.B. Apthorpe, 2011; die Beiträge in Fechter und Hindman, 2011 und in Mosse, 2011a; Harrison, 2013; Stirrat, 2008). Aufgrund seiner (auto-)ethnographischen Perspektive wird er oft als ,,aidnography“ (Gould, 2004) betitelt und stellt ,the social and cultural lifes of global professionals themselves, their class position, biographies, commitments and anxieties" (Mosse, 2011b: 14) in den Fokus. Viele Autor*innen dieser Forschungsrichtung waren selbst in der Hilfs- und Entwicklungsindustrie tätig und machen ihre eigenen Erfahrungen zum Gegenstand der Forschung.
In diesem Punkt liegt einerseits ein großer empirischer Vorteil, gleichzeitig aber auch ein gewisses Problem: Einige Arbeiten lassen sich stark vom empirischen Material und von der ethnographischen Beobachtung leiten und entsprechen damit eher einer dichten Beschreibung des Erlebten und einer intensiven Reflexion der eigenen Rolle (vgl. Korf, 2004: 220), auch in Bezug auf ungleiche Machtverhältnisse (vgl. z.B. Shutt, 2006). Was in Teilen der Debatte bisher zu kurz kommt, ist eine machtkritische, theoretischkonzeptionelle Fundierung der Forschung und eine Rückbindung der Selbstreflexion an Konzepte, die die Konstitution des Subjekts, identitäre Spannungen, Polyphonien und Widersprüche in den Blick nehmen.

Vor diesem Hintergrund knüpft dieser Beitrag zwar an das Thema der Aidnography an, verschränkt es aber mit einem poststrukturalistischen Konzept von Subjektivität. Dieses berücksichtigt, dass Subjekte nicht als einheitliche, autonome Individuen gegeben sind, sondern in komplexen und machtvollen historischen und sozialen Prozessen hervorgebracht und durch diskursive Praktiken reproduziert und transformiert werden (vgl. z. B. Bröckling, 2007). In diesem Verständnis eignen sich insbesondere gouvernementalitätstheoretische Ansätze, um den Fokus auf fragmentierte Subjekte und ihre konfligierenden Subjektpositionen zu schärfen und damit das Verhältnis zwischen „Entwicklung“ als hegemonialem Konzept und den Subjektivierungsprozessen von Entwicklungspraktiker*innen auszuloten.

Im Folgenden zeigt Kap. 2 zunächst, wie Gouvernementalitätsansätze im Anschluss an Michel Foucault das Spannungsfeld zwischen kollektiven Diskursen der „Entwicklung" und eigenen Praxiserfahrungen sowie Subjektivierungsprozesse erklären können. Nach dieser Annäherung an eine poststrukturalistische Interpretation von Subjektivität werden in Kap. 3 die empirische Grundlage des Beitrages und das methodische Vorgehen erläutert. Kapitel 4 widmet sich den empirischen Ergebnissen und zeigt auf, über welche Mechanismen sich das diskursive Ordnungsprinzip der „Entwicklung“ in den Identitätskonstruktionen der Entwicklungspraktiker*innen niederschlägt (Kap. 4.1) und wie sich praktische Erfahrungen, (Selbst-)Zweifel und identitäre Fragmentierungen auf ihre Subjektivierungsprozesse auswirken (Kap. 4.2.1). Im Anschluss fragt der Beitrag, inwiefern eine Dissonanz zwischen dem Imperativ der „Entwicklung“ und einer selbstreflexiv-kritischen Haltung Möglichkeiten eröffnet, die Entwicklungspraxis anzueignen, herauszufordern und zu transformieren (Kap. 4.2.2). Er endet mit einem Fazit und einem Ausblick (Kap. 5).

\section{Die Perspektive der Gouvernementalität}

Gouvernementalitätstheoretische Ansätze im Anschluss an Michel Foucault (insb. 2006a [1978]; 2006b [1979]) und ihre Weiterentwicklungen durch die Governmentality Studies (vgl. als Vorreiter Burchell et al., 1991; für einen Überblick 
über die Rezeptionsgeschichte vgl. z. B. Bröckling et al., 2000; Dean, 2010; Lemke, 2007; Walters, 2012) legen ein besonderes Augenmerk auf Machteffekte und unterschiedliche Regierungsweisen. Sie verstehen Macht und Regierung nicht allein als repressive, staatlich-institutionalisierte Kategorien, sondern machen auf subtile Machtbeziehungen in allen Formen gesellschaftlicher Interaktion aufmerksam (vgl. Foucault, 1987 [1983], 1983 [1976]). In diesem breiten Machtverständnis kommt dem Aspekt der (Selbst-)Führung eine wichtige Rolle zu. Er richtet den analytischen Blick auf die Prozesse, in denen sich Menschen nicht allein durch äußeren Zwang oder Gesetze, sondern durch verinnerlichte Normen und Werte sowie gesellschaftliche Verantwortungsdiskurse in bestehende Deutungsangebote des Selbst einfügen und sich als Subjekte identifizieren (vgl. Linnemann, 2018: 5).

Mit diesem konzeptionellen Fokus auf Formen der Subjektivierung und (Selbst-)Führung hat die Perspektive der Gouvernementalität in den letzten Jahren auch vermehrt Eingang in die Humangeographie gefunden. Insbesondere Fragen des Städtischen (vgl. z.B. Dzudzek, 2016; Mattissek, 2008; Rosol, 2015; Schipper, 2013; Raco und Imrie 2000; Füller und Marquardt, 2010), der Wohnungslosigkeit (z.B. Marquardt, 2013), Integration (z.B. Pütz und Rodatz, 2013) und Migration (z.B. D'Aoust, 2013; Tazzioli, 2014) werden zunehmend durch die Brille der Gouvernementalität gelesen.

Bisher weniger Beachtung hat Foucaults Konzept der contre-conduite gefunden (vgl. z.B. Cadman, 2010; Davidson, 2011; Death, 2010; Linnemann, 2018; Rosol, 2014 und die Beiträge in Odysseos et al., 2016). Damit ergänzt er seine Überlegungen zu Macht und Führung durch widerständige, die hegemoniale Ordnung herausfordernde Praktiken (Foucault, 2006a [1978]: 292ff.) und bietet so auch für diesen Beitrag ein vielversprechendes Konzept, um die Aneignung und Transformation der Entwicklungspraxis zu analysieren.

Das Konzept der contre-conduite schließt implizit an Foucaults relationales Verständnis von Macht an und betont ihre produktive Seite, die Veränderungen und Verschiebungen der Ordnung auch und gerade im Kleinen ermöglicht:

„Große radikale Brüche, massive Zweiteilungen? So was kommt vor. Aber weit häufiger hat man es mit mobilen und transitorischen Widerstandspunkten zu tun, die sich verschiebende Spaltungen in eine Gesellschaft einführen, Einheiten zerbrechen und Umgruppierungen hervorrufen, die Individuen selber durchkreuzen, zerschneiden und umgestalten, in ihrem Körper und in ihrer Seele abgeschlossene Bezirke abstecken. [...] [S]o streut sich die Aussaat der Widerstandspunkte quer durch die gesellschaftlichen Schichtungen und die individuellen Einheiten“ (Foucault, 1983 [1976]: 96f.).

Durch den Bezug auf das Konzept der Führung bezieht der Begriff auch diffuse, sanfte Widerstandsformen mit ein und rückt den Prozess der Subjektivierung mit all seinen Brüchen und Widersprüchen in den Vordergrund (vgl. Foucault, 2006a [1978]: 282ff.). Außerdem wird er der Suche nach einer anderen Form der Führung gerecht, nach einem „Andersgeführt-werden, durch andere Menschen, auf andere Ziele hin“ (Foucault, 2006a [1978]: 288). Widerständige Praktiken werden auf diese Weise nicht als binäres Gegenüber einer hegemonialen Ordnung gedacht, sondern als iterative Momente, die in Form einer ,Grenzhaltung“ (Foucault, 1990: 48, zitiert nach Opitz, 2004: 86) Bedeutungen und Regeln verschieben. Auf diese Weise zeigt das Konzept der contreconduite, dass einfache Dichotomien zwischen Macht und Widerstand, dafür und dagegen, nicht nur zu kurz greifen, sondern auch Alternativen im Dazwischen verdecken (vgl. auch Gibson-Graham, 2006a [1996], 2006b).

In diesem Sinne lotet der Beitrag aus, welchen Mehrwert das Konzept der contre-conduite für die Analyse von widerständigen Praktiken und politischen Interventionen bieten kann, die die Entwicklungspraxis im Kleinen herausfordern und aneignen, ohne losgelöst davon zu funktionieren. Zunächst widmet sich das folgende Kapitel den Ergebnissen der Analyse von (Selbst-)Führungsmechanismen und identitären Fragmentierungen, um sich abschließend auf eine Spurensuche nach Formen der contre-conduite im Feld der „Entwicklungszusammenarbeit" zu begeben.

\section{Empirische Grundlage und methodisches Vorgehen}

Die empirische Grundlage des Beitrages bildet ein qualitatives und exploratives Untersuchungsdesign aus 22 narrativbiographischen, teilweise leitfadengestützten Interviews mit Menschen, die im nichtstaatlichen Bereich der „Entwicklungszusammenarbeit“ und „Humanitären Hilfe“ tätig sind oder waren ${ }^{2}$. Die offene und tiefgründige Form narrativbiographischer Interviews eröffnet einen Raum für dichte und möglichst ungestörte Erzählpassagen, in denen detaillierte Beschreibungen von Arbeitsalltag, Erlebnissen und Gefühlen sowie Reflexionen über die eigene Arbeit, ihren Sinn und persönliche Werte möglich sind (vgl. Przyborski und Wohlrab-Sahr, 2014: 126ff.). Der Fokus liegt damit anders

\footnotetext{
${ }^{2}$ Die Konzentration auf nichtstaatliche Organisationen geht aus der Vorläuferstudie „Die Geopolitik der Hilfsbedürftigkeit“" (gefördert von der Fritz Thyssen Stiftung 2011-2013) hervor, in der die Spendenwerbung nichtstaatlicher Organisationen diskursanalytisch untersucht worden ist (vgl. Linnemann und Reuber, 2015). Bei der Auswahl der Gesprächspartner*innen wurde auf ein ausgeglichenes Verhältnis hinsichtlich Gender, Alter, Arbeitserfahrung und der Position innerhalb der Organisationen geachtet. Das Sample umfasst (ehemalige) Mitarbeiter*innen der Organisationen Brot für die Welt, Misereor und Welthungerhilfe (sowie Fachkräfte, die von diesen Organisationen über das deutsche Entwicklungshelfer-Gesetz (EhfG) an Partnerorganisationen im Ausland entsandt wurden), Johanniter Unfall-Hilfe, Diakonie Katastrophenhilfe sowie der deutschen Sektionen von Oxfam und Ärzte ohne Grenzen.
} 
als im Bereich der Aidnography nicht auf einer ethnographischen Begleitung der Personen in ihrem Arbeitsalltag, sondern auf der Analyse ihrer versprachlichten Einschätzungen, Bewertungen und Reflexionen ihrer alltäglichen Arbeit.

Die Auswertung der Interviews stützt sich auf aussagenanalytische Mikromethoden der Diskursforschung (Angermüller, 2007; Glasze, 2007; Mattissek, 2008, 2009). Diese sprachwissenschaftlichen Verfahren untersuchen, wie in einzelnen Textpassagen Bedeutung hergestellt wird und welchen Regeln diese Sinnproduktion folgt. Um die Herstellung von Bedeutung herauszuarbeiten, konzentrieren sich die Mikroverfahren der Aussagenanalyse u.a. auf Pronomen und Konnektoren, die einen Bezug zum Kontext der Aussage herstellen, und damit auf implizite Elemente und (Un-)Regelmäßigkeiten hegemonialer diskursiver Ordnungen verweisen (vgl. Mattissek, 2009: 279ff.):

Die Analyse deiktischer Formen wie ,ich“, „du“, „,hier“, „dort“ oder ,,jetzt“, ,früher“ gibt Aufschluss darüber, wie das Eigene und das Fremde sprachlich getrennt und mit Bedeutungen verknüpft wird und lässt Rückschlüsse auf die sprachliche Herstellung von Subjektivität und Identität zu (vgl. Mattissek, 2009: 284).

Polyphone Markierungen weisen auf Subtexte, Widersprüche, Konflikte und implizit vorausgesetzte Sichtweisen hin, die durch Verneinungen, Ironie und Konnektoren wie „,nicht“, ,,jedoch“, ,aber“ und „,sondern“ auf unterschiedliche Distanz gehalten werden. Die Analyse der polyphonen Struktur von Aussagen eignet sich darum besonders zur Untersuchung identitärer Brüchigkeit, Heterogenität und Widersprüchlichkeit (vgl. Ducrot, 1984; Mattissek, 2008: 138ff.).

Vorkonstrukte, wie z.B. nicht-notwendige Relativsätze und Nominalisierungen (z.B. entwickeln $\rightarrow$ Entwicklung), verweisen auf ein diskursives Vorwissen, in das eine Aussage eingebettet ist, und lassen Rückschlüsse auf Wertungen und Positionierungen im Hintergrund der Aussage zu (vgl. Pêcheux, 1983 [1975]; Mattissek, 2008: 137; Angermüller, 2007: 154).

Mit Hilfe dieser Mikroverfahren und dem Fokus auf die Verbindung von Text und Kontext lässt sich anhand des Interviewmaterials die wechselseitige Beziehung zwischen Diskursen der „Entwicklung“ und Identitätskonstruktionen von Entwicklungspraktiker*innen rekonstruieren. Insbesondere die Analyse polyphoner Strukturen in Verbindung mit deiktischen Partikeln kann wertvolle Einblicke in die Brüchigkeit und Heterogenität von Subjektivierungsprozessen geben, die bei einem alleinigen Fokus auf den Inhalt der Aussage im Verborgenen bleiben.

\section{Entwicklungspraktiker*innen zwischen Selbstführung, Fragmentierung und contre-conduite}

Der verbleibende Teil des Beitrages widmet sich nun dem Interviewmaterial und zeigt auf, wie mit Hilfe der vorge- stellten Mikromethoden einerseits Subjektivierungsprozesse nachgezeichnet, aber auch subjektive Fragmentierungen und Ambivalenzen analysiert werden können. Dabei geht es nicht in erster Linie um die Darstellung individueller Perspektiven, Erfahrungen und Lebensläufe. Im Sinne der gouvernementalitätstheoretischen Perspektive der Analyse sollen vielmehr überindividuelle Muster der Subjektivierung, (Selbst-)Führung und des Umgangs mit identitären Fragmentierungen herausgearbeitet werden.

\subsection{Selbstführung und Selbstoptimierung im Sinne des "Guten"}

Mit Blick auf Beweggründe und Motivationen ist es zunächst wenig überraschend, dass sich der Diskurs der „Entwicklung" auch in den Subjektivierungsprozessen der Entwicklungspraktiker*innen widerspiegelt. Ihre Motivation besteht darin, anderen ,etwas Gutes tun zu wollen“ (I-01: 38) ${ }^{3}$, an „etwas Größerem“ (I-16: 10) oder „,ür die gute Sache“ (I04: 60) zu arbeiten, einen positiven Beitrag zu leisten und Verbesserungen zu erreichen (vgl. auch Ruwanpura und Hollenbach, 2014). Einige der Befragten sprechen von ihrer „Bestimmung" (I-22: 10) und fühlen sich - wie im Titel dieses Beitrages - regelrecht zu ihrer Aufgabe berufen (I-10: 24).

Ein genauerer Blick auf den inneren Antrieb Gutes zu tun zeigt, dass sich der Entwicklungsdiskurs mittelbar in Form verschiedener Ideale und Überzeugungen in den Subjektkonzeptionen niederschlägt. Neben Werten wie Toleranz, Respekt und Offenheit spielen vor allem verinnerlichte politische und rechtebasierte Ideale eine bedeutende Rolle für die Motivation der Entwicklungspraktiker*innen, was folgendes Zitat beispielhaft verdeutlicht: ${ }^{4}$

„Armut, [...] politische Ungerechtigkeit, Menschenrechtsfragen, Demokratisierung, das waren Themen, die mich stark beschäftigt haben. Und zu denen es mich hingezogen hat. [...] Demokratisierungs- und Gerechtigkeitsfragen haben mich stark angetrieben." (I-06: 15-21)

Der Entwicklungsdiskurs wird in Form einzelner Werte und Motivationen verinnerlicht und in eine wertegeleitete Selbstführung übersetzt. Viele Nominalisierungen wie „Ungerechtigkeit“ und „Demokratisierung“" verweisen auf eine diskursiv gefestigte, normative Referenzlinie im Hintergrund

\footnotetext{
${ }^{3}$ Die anonymisierten Quellenangaben von Interviewzitaten folgen einer zufälligen Nummerierung der Interviews (I-01 bis I-22) und der Angabe der Absätze im jeweiligen Transkript, denen die zitierte Aussage entnommen wurde.

${ }^{4}$ In den Interviewzitaten werden folgende aussagenanalytische Markierungen vorgenommen: Deiktische Partikel $=$ fett, Polyphonie $=$ unterstrichen, Vorkonstrukte $=$ kursiv, Pausen werden je nach Länge mit (.), (..) oder (...) gekennzeichnet, wobei (.) für eine Sekunde steht. Auslassungen werden durch [...] angezeigt. Für die Anonymisierung veränderte Angaben werden mit \# gekennzeichnet.
} 
der Aussage: Durch die eigene Idee eines humanistischabendländisch geprägten „Guten“ fühlt sich die Person ,stark angetrieben“; sie fügt sich demnach durch internalisierte normative Positionen und Wertvorstellungen in gesellschaftlichdiskursiv hergestellte Deutungsangebote des Selbst ein.

Ein wichtiger Antrieb der Selbstregierung und ein Ankerpunkt der Identifikation ist die Sinnhaftigkeit der Arbeit bezogen auf die eigenen Werte. Das folgende Zitat zeigt eindrücklich, wie über den häufigen Gebrauch der deiktischen Partikel ,ich“ und ,wir“ ein sehr enger Bezug zur Arbeit hergestellt und der persönlichen Überzeugung großes Gewicht verliehen wird:

„Ich glaube es nicht nur, sondern ich bin überzeugt davon, dass ich eine sehr sinnvolle Arbeit mache. Ich hoffe sehr, dass ich dazu beitrage [...], dass sich gewisse Dinge in der Gesellschaft verändern, vielleicht auch politisch verändern, dass wir zumindest einen Push geben.“"(I-14: 67)

Die Selbstführung speist sich aber nicht nur aus der internalisierten Logik, ,Gutes“ zu tun, sondern gleichzeitig auch aus der Suche nach Sinnhaftigkeit, persönlicher Befriedigung und Selbstverwirklichung: „Man bekommt, wenn man was Gutes tut, ein gutes Gefühl zurück“ (I-01: 38). Ein häufiges Muster ist in diesem Zusammenhang die Abgrenzung von früheren Tätigkeiten und der Privatwirtschaft:

„Die Befriedigung, die ich hatte, wenn ich mein Gehalt als Steuerfachangestellter bekommen habe oder wenn ein Kunde sich dafür bedankt hat, dass die Steuerrückzahlung hoch war, die ist nicht mit der Befriedigung zu vergleichen, die ich habe, wenn ich hier sehe, dass junge Menschen ausgebildet werden oder Felder auf eine Art und Weise bewirtschaftet werden, dass es mehr Erträge gibt. Ich empfinde das als sehr befriedigend hier zu arbeiten. Man hat das Gefühl, dass es Sinn hat." (I20: 25)

Diese Aussage weist exemplarisch auf einen spezifischen Aspekt der Selbstführung in der Entwicklungspraxis im Vergleich zu vielen anderen Berufsfeldern hin: Ähnlich wie in anderen sozialen und medizinisch-pflegerischen Berufen wird der Sinn der entwicklungspraktischen Arbeit moralisch hoch bewertet, normativ aufgeladen und idealisiert; er geht weit über das schlichte „Geldverdienen“ hinaus (vgl. de Jong, 2011: 31; Bronner, 2003: 156).

In Form von ausgeprägter Kritik an der Lebensweise des globalen Nordens und (neo-)kolonialen Abhängigkeitsverhältnissen kommt im Bereich der Entwicklungspraxis ein weiteres Charakteristikum hinzu, das eine zusätzliche Triebfeder der Selbstführung bildet. Über Vorkonstrukte und Nominalisierungen wie ,Verantwortung“, „SDGs“ (Sustainable Development Goals) und „Globalisierung“ knüpfen die folgenden, postkolonial-kritischen Aussagen an sozialökologische Verantwortungsdiskurse von Nachhaltigkeit und Postwachstum an. Auf diese Weise aktivieren sie Selbstführungslogiken und appellieren an das Gewissen der Subjekte (vgl. Linnemann, 2018):

„Wir müssen immer mehr in einen Umkehrprozess kommen. Globalisierung muss bei uns stattfinden, die SDGs zielen auch darauf ab, dass sich bei uns (betont) etwas ändert, wir müssen unseren Lebensstil ändern, wir müssen uns da ganz neu aufstellen." (I-06: 55)

„Man kann nicht eine Welt verändern und niemand muss dafür bluten, sondern wir müssen alle dazu beitragen, den Lebensstil zu ändern und das geht eben nicht einfach immer schneller, doller, weiter, dann ist die Welt irgendwann kaputt.“ (I-18: 25)

Zur Idealisierung der Arbeit gesellt sich demnach häufig das persönliche Verantwortungsgefühl, mit dem eigenen Lebensstil und den Privilegien des globalen Nordens für die Probleme derer mitverantwortlich zu sein, denen „Gutes“ getan werden soll. Diese Kombination aus internalisierten Verantwortungsdiskursen und normativer Aufladung der Arbeit ist charakteristisch für die Ausbildung von Selbstführungslogiken in der Entwicklungspraxis.

Hinter allen Ausprägungen dieser Selbstführung im Sinne der ,guten Sache“ steht das grundlegende Prinzip der Selbstoptimierung. Dabei sind die formalen Parallelen zur Selbstführung neoliberaler Prägung (vgl. Bröckling, 2007) groß, es unterscheiden sich allein die Grundwerte: Anstelle eines ökonomistischen Referenzrahmens steht das ethischnormative Prinzip des „Guten“ mit dem Ergebnis eines „entwicklungsunternehmerischen Selbst“ (in Anlehnung an Bröckling, 2007), das sich den internalisierten und als gut gesetzten westlich-humanistischen Grundwerten verpflichtet: „Gute Arbeit" leisten wollen (I-08: 33), Fehler ausmerzen und das „Beste rausholen“ (I-03: 21), ständig dazulernen und sich weiterentwickeln, Herausforderungen suchen und „flexibel bleiben“ (I-05: 56), sowie die Frage der Relevanz und Effizienz der eigenen Arbeit bilden einen Kreislauf der Selbstoptimierung. Diese wird angetrieben durch die wertegeleitete Verpflichtung gegenüber der ,guten Sache“, die als Motor im Kern des Kreises steht. Abbildung 1 veranschaulicht die einzelnen Stationen dieses Kreislaufs der Selbstoptimierung durch weitere beispielhafte Interviewzitate.

Neben solchen Reflexionen über die eigene Selbstoptimierung berichten viele der Interviewpartner*innen über Selbstoptimierung vom Hörensagen und Beobachtungen von Kolleg*innen kurz vor der Selbstaufgabe für die Arbeit. Oftmals schwingt in diesen Berichten implizit Kritik an der Logik der Selbstoptimierung mit:

„Ich glaube, dass da einige NGOs [...] ganz schön drunter leiden, diesen Druck, dass alles [Geld, 


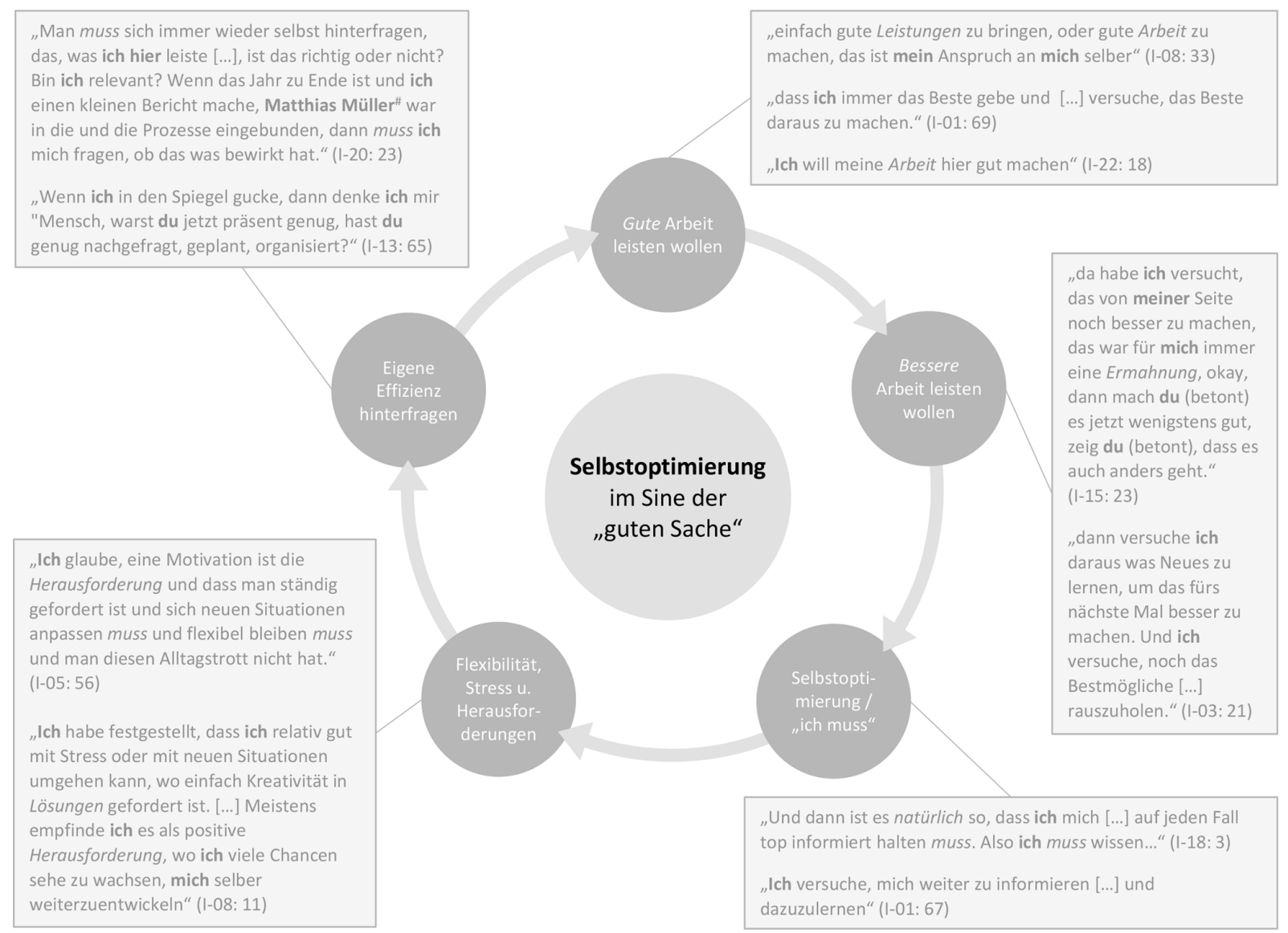

Abb. 1. Formen der Selbstoptimierung im Sinne der „guten Sache“ ("Name geändert). Quelle: Eigene Darstellung.

Anm. d. A.] in die Projekte gehen muss und dadurch von den Mitarbeiterinnen und Mitarbeitern sehr, sehr viel abverlangt wird. Mehr als vielleicht in anderen Branchen. [...] Ich habe viele Leute erlebt, die weit über ihrem gesunden Arbeitspensum arbeiten. (..) Auch, weil sie das Gefühl haben, sie müssen ja irgendwo, weil es ist ja für was Gutes.“ (I-16: 48)

Dieses Zitat zeigt eindrücklich, wie der strukturelle Effizienzdruck der Organisationen an die Mitarbeiter*innen weitergegeben wird. So schreibt auch Hindman, ,[that the] pursuit of efficiency has found a new place in the development world such that ,best practices ' are not only applied by aid workers but applied to them as well" (2011: 177, Herv. i. Orig.). In den aid workers trifft die Logik der Effizienz auf einen idealen Nährboden, nämlich auf die internalisierten Verantwortungsdiskurse und das Ideal „Gutes“ zu tun. Gemeinsam mit der Logik der Selbstführung im Sinne des „Guten" potenziert sie sich in einigen Fällen bis hin zur Selbstüberschätzung, Selbstaufgabe und im Äußersten zum Burnout (vgl. I-05: 114). Auf ähnliche Weise beschreibt Bronner (2003: 156f.) im Rahmen einer psychologischen Studie zu humanitären Helfer*innen in Krisengebieten, dass mit zunehmend idealistischer Arbeitsmotivation das Risiko von Burnout-Erscheinungen steigt: „Kurzum, der idealistischen Überhöhung der Arbeit folgt die Ernüchterung" (Bronner, 2003: 157; zum Zusammenhang von (neoliberaler) Selbstverwirklichung und Burnout vgl. Roth, 2015: 57ff.).

Dieser innere Druck schafft identitäre Widersprüche und brüchige, fragmentierte Subjekte, die zwischen verschiedenen Identitätsanteilen und Subjektpositionen changieren. Das folgende Kapitel wendet sich darum im Sinne eines poststrukturalistischen Verständnisses von Identität diesen Heterogenitäten und inneren Fragmentierungen zu.

\subsection{Der Umgang mit (Selbst-)Zweifeln und identitären Fragmentierungen}

Trotz - oder gerade wegen - der machtvollen Selbstführungslogiken im Sinne der ,guten Sache“ haben viele Gesprächspartner*innen auch von Momenten der Frustration, Desillusionierungen und (Selbst-)Zweifeln in ihrem Arbeitsleben berichtet. Neben individuellen Fragmentierungslinien zwischen privaten und beruflichen Meinungen und in Bezug auf die (Un-)Vereinbarkeit von Familienleben und Jobs im Ausland werden Frustrationen und Zweifel meistens zu- 
nächst in Bezug auf bürokratische Strukturen und interne Arbeitsabläufe geäußert. Dahinter lauern aber in vielen Fällen auch tiefe Sinn- und Legitimationsfragen in Bezug auf Inhalt, Ziel und Zweck der Arbeit, die häufig zu identitären Fragmentierungen und Widersprüchen führen:

„Ich erinnere mich an eine Zeit in Burundi" , [...] wo ich viel erlebt habe, viel gesehen habe, wo ich dachte, mein Gott, was soll dieser Scheiß, den wir hier alle machen?" (I-15: 21)

Neben generellen Sinnfragen wie diesen wird häufig die konkrete Wirkung der eigenen Arbeit kritisch hinterfragt (vgl. Kap. $4.1 \mathrm{zu}$ Selbstoptimierung) und die Legitimation von ,EZ“ allgemein infrage gestellt:

„Meine Organisation" will nicht der Ausputzer für die großen Sünden sein, für Fehlverhalten von einer falschen Wirtschaftspolitik, die immer mehr Arme produziert. ,Es gibt ja die Hilfswerke, die das Elend lindern. 'Das kann's ja nicht sein. Das macht mich wütend, wie hilflos man dann manchmal ist. Dann hat man gerade mühevoll eine Perspektive für Jugendliche durch Ausbildungszentren aufgebaut und dann kommen irgendwelche Billigimporte von Fernost und machen das alles kaputt. Das sind natürlich konkrete Rückschläge in der Arbeit, die man ständig erlebt.“ (I-18: 23)

„Ich stelle mir auch die Frage, ob das nicht ein bisschen anmaßend ist [...], zu behaupten, wir wüssten besser, was für die Menschen hier vor Ort unter ihren Lebensbedingungen besser wäre. Und vielleicht wissen wir das gar nicht besser. Dass es hier Missstände gibt, ist selbstverständlich, [...] aber wer sind wir, um den Menschen zu sagen, wie sie es richtiger machen sollten?" (I-01: 65)

Sinn- und Legitimationsfragen wie diese lösen in vielen Fällen Wut, Frustration und Enttäuschung aus. Angesichts der ethisch-normativen Prinzipien der „Entwicklungsarbeit“ und der beschriebenen Selbstführungslogiken ist die Frage nach Umgangsweisen mit diesen Sinnfragen und Widersprüchlichkeiten von besonderem Interesse (vgl. auch Korf, 2018: 159f.).

Die beobachteten Umgangsweisen mit Verstörungen, Desillusionierungen und Frustrationen im Laufe der Karriere nehmen sehr unterschiedliche Ausprägungen an, die sich didaktisch zugespitzt auf einer kontingenten Skala mit zwei Polen abbilden lassen. Während der erste Pol verschiedene Umgangsweisen repräsentiert, die Zweifel und Sinnfragen konsequent einzuhegen und diskursiv zu glätten versuchen (Kap. 4.2.1), steht der zweite Pol für das Offenhalten von identitären Brüchen und eine kritische Distanz, die einen Raum für widerständige Praktiken eröffnet (Kap. 4.2.2). An beiden Polen wirken jedoch (unterschiedliche) Logiken der Selbstführung mit dem Ziel, die Sinnhaftigkeit des eigenen Tuns argumentativ aufrechtzuerhalten oder wiederherzustellen.

\subsubsection{Identitäre Narrationen}

Die Einhegung von Zweifeln und Sinnfragen am ersten Pol folgt der modernen Vorstellung einer stabilen, monolithischen Identität der (von mir als solche angerufenen) Subjekte. Sie versuchen in vielen Fällen, ihre divergierenden Identitätsanteile rhetorisch unter einen Hut zu bringen, Zweifel auszublenden und insgesamt eine logische Geschichte über ihr eigenes Ich zu erzählen:

„Das war für mich ein sehr langer Prozess und mit viel Reflexion und Kritik und Hinterfragen verbunden, bis man dann für sich selber persönlich Entschlüsse gefasst hat, das ist jetzt in Ordnung." (I02: 20)

Die Narration einer kohärenten Identität ist Teil vieler Subjektivierungsprozesse. Gleichzeitig ist sie das Ergebnis verschiedener Umgangsformen mit identitären Fragmentierungen und Verschiebungen, die mit Beispielzitaten in Abb. 2 dargestellt sind, um die Unterschiedlichkeit und Vielfalt der Umgangsweisen und Subjektivierungsprozesse plastisch hervorzuheben. Die gewählten Kategorien lassen sich jedoch nicht trennscharf voneinander abgrenzen, sondern sind durch Überschneidungen und fließende Übergänge gekennzeichnet.

\section{Prinzip „Hoffnung und Überzeugung}

Es scheint nur wenig überraschend, dass der Knotenpunkt vieler Identitätsnarrationen in der inkorporierten Logik des „Guten“ und dem festen Glauben an den tieferen Sinn der eigenen Arbeit begründet liegt. Die internalisierten Verantwortungsdiskurse führen nicht nur zu einer Selbstführung im Sinne der „guten Sache“, sondern befähigen das „,entwicklungsunternehmerische Selbst“ darüber hinaus, Desillusionierungen und andere identitäre Fragmentierungen zu glätten und ,seinen Frust [zu] bewirtschaften“ (I-12: 26).

Dies wird am Beispiel einer Person deutlich, die immer wieder kritisch und nachdenklich über Schwierigkeiten und Enttäuschungen im Laufe ihrer Karriere berichtet, diese aber sogleich im nächsten Satz nivelliert und über polyphone Satzstrukturen in den Schatten ihrer tiefen Überzeugung stellt:

„Ach, ich habe gehofft, dass durch Entwicklungszusammenarbeit mehr Räder in Bewegung geraten. Und zwar auch, wenn man in einem Projekt arbeitet zur Hühner" ${ }^{\#}$ haltung. Und habe dann aber begreifen müssen, dass, kurz danach gab es in Ruanda $^{\#}$ diesen Genozid, den Krieg, und damit war 


\section{Die Einhegung von Zweifeln und Sinnfragen}

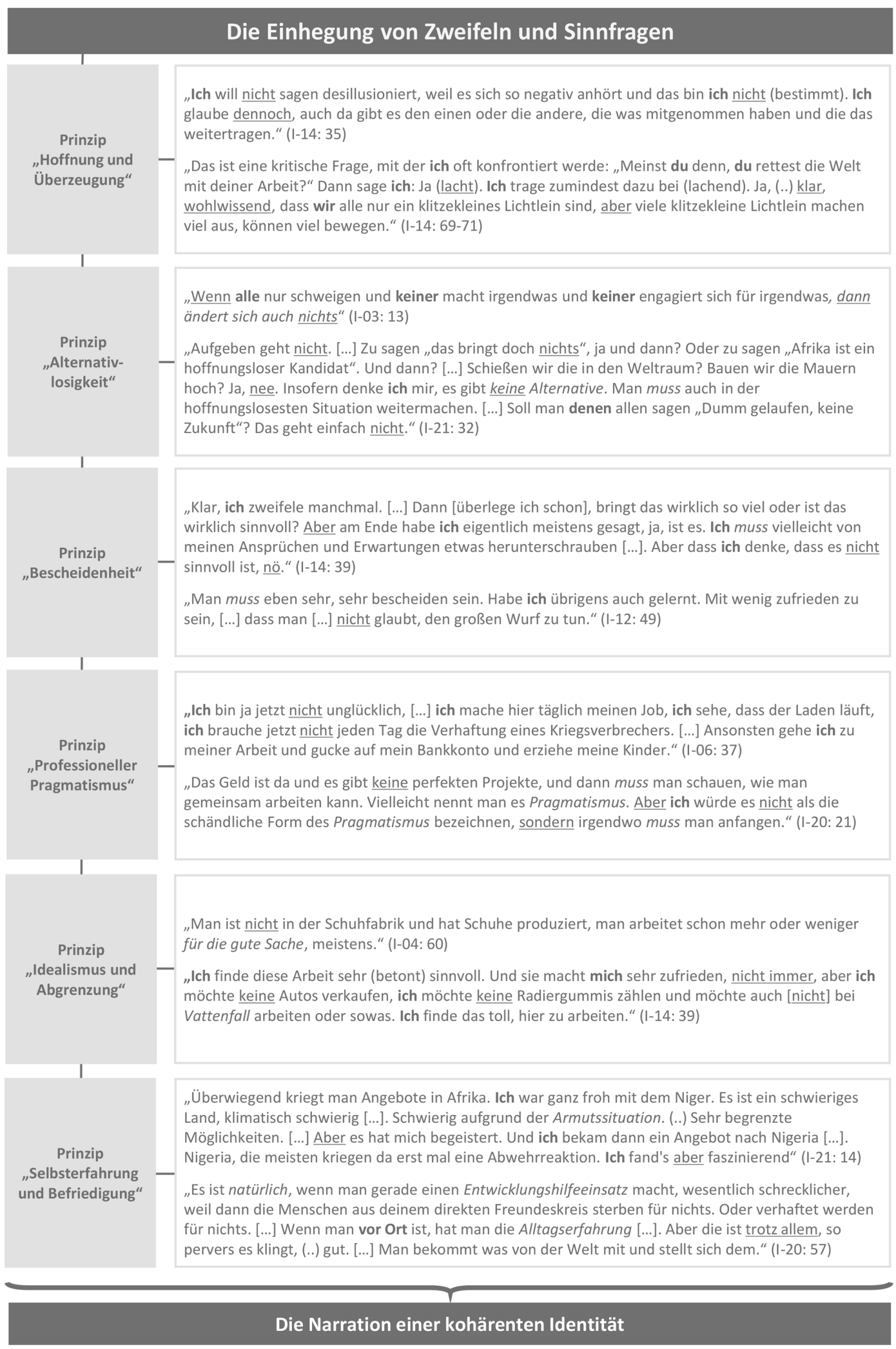

Abb. 2. Die narrative Einhegung von Zweifeln und Fragmentierungen. Die didaktische Systematisierung in sechs Kategorien dient der Hervorhebung der Unterschiedlichkeit und Vielfalt der Umgangsweisen und Subjektivierungsprozesse. Die Übergänge zwischen den Kategorien sind jedoch fließend und nicht trennscharf. Quelle: Eigene Darstellung. 
alles platt. Alles, was wir in Jaaahren [...] aufgebaut hatten, war zerstört. Den Menschen ging es nur noch ums Überleben, die haben nicht geguckt, welches Huhn ${ }^{\#}$ [sie] mit was ankreuzen. Und die Menschen, die wir geschult hatten, die lebten zum Großteil gar nicht mehr. Also es muss auch nicht immer so hart sein, aber da ist mir schon bewusst geworden, wie wenig wir letztendlich wirklich erreichen können (atmet aus) (..). Also, (hebt die Stimme, lauter) ich will nicht sagen desillusioniert, weil es sich schon wieder so negativ anhört und das bin ich nicht (bestimmt). Ich glaube dennoch, auch da gibt es vielleicht den einen oder die andere, die was mitgenommen haben und die das weitertragen."(I-14: 35)

Über zahlreiche deiktische Partikel schafft sie einen persönlichen Bezug zu ihren Aussagen und eine enge identitäre Verknüpfung zwischen sich und ihrer Arbeit. Ähnlich wie in diesem Beispiel berichtet sie auch sehr offen über persönliche Enttäuschungen und Gefühle in Verbindung mit Korruption in den Partnerländern. Auch hier zeugt die deiktische Partikel ,wir" von einer starken Identifikation mit ihrem Tun, insbesondere in ihrer Kontrastierung zu ,der Politik“ und denen, „die in der Regierung sitzen“. Durch die über den Konnektor ,nichtsdestotrotz“ markierte Polyphonie rückt ihre Traurigkeit jedoch in den Hintergrund der internalisierten Verantwortung, ,die Leute zu unterstützen“:

„Gerade, wenn die Politik selbst sich die Taschen füllt [...]. Wo wir dann sagen, meine Güte, wir versuchen, von unten was zu verändern und es tut sich nichts, weil da oben, die in der Regierung sitzen, die interessiert es nicht, dass ihre eigene Bevölkerung verhungert. Denen ist das völlig egal (betont). Und sowas ist dann schon echt traurig mitanzusehen. Nichtsdestotrotz ist es wichtig, die

Leute zu unterstützen."(I-14: 35)

Auf diese Weise räumt sie tieferliegenden Zweifeln wenig Platz ein und erschafft eine lineare Erzählung ihrer eigenen Identität und des Sinns ihrer Arbeit vor dem Hintergrund der ,guten Sache“.

\section{Prinzip „Professioneller Pragmatismus“}

Neben dieser Form der Identitätsnarration auf der Grundlage von Hoffnungen, Überzeugungen und dem tiefen Glauben an den Sinn der Arbeit stellt das Kitten von Brüchen durch einen professionellen Pragmatismus eine andere Umgangsweise dar. Diese wird beispielhaft an den Aussagen einer Person deutlich, die nach entwicklungsbezogenen Auslandstätigkeiten nun seit einigen Jahren in einer Organisation in Deutschland arbeitet. In ihren Aussagen ringen immer wieder idealistische, normative Selbstführungslogiken im Sinne der ,guten Sache“ mit pragmatischen Subjektanteilen und
Entscheidungen im Sinne von Karriere und Familie. So antwortet sie auf die Frage nach ihrem persönlichen Umgang mit Zweifeln und Frustrationsmomenten:

„Gut, man professionalisiert sich natürlich, man wird älter, man kriegt Kinder, irgendwann macht man seinen Job sozusagen. Das heißt jetzt nicht, dass ich nicht mehr motiviert bin, aber ich mache jetzt hier auch einen ganz anderen Job als damals noch in Simbabwe ${ }^{\#}$ [...]. Ich glaube nicht, dass ich weniger idealistisch bin, aber die Erwartungshaltung ist nicht mehr so hoch, sondern man muss halt jetzt gucken, was man realistisch machen kann, [...] vielleicht wird man auch einfach professioneller. Aber, klar, dieses naive ,Jetzt organisieren wir die Revolution und dann wird alles gut' (lachend), das schleift sich natürlich ab." (I06: 25)

In dieser Aussage wird durch polyphone Satzstrukturen anschaulich, wie unterschiedliche Subjektpositionen in einem fragmentierten Subjekt nebeneinander existieren und im Prozess der Subjektivierung um die Deutungshoheit ringen. Die idealistischen Subjektanteile werden in diesem Abschnitt zum Teil durch die polyphonen Marker ,aber“ und ,sondern“ im Hintergrund gehalten, während die professionell-pragmatischen Stimmen die Aussage dominieren, die Zweifel auf Abstand halten und frühere Erwartungshaltungen als „naiv“ beiseitedrängen. Dieser erste Eindruck verschiebt sich jedoch mit einem genaueren Blick auf die unterschiedliche Verwendung der Pronomen ,ich“ und „man“: Die unpersönliche deiktische Partikel „man“ tritt wie z.B. im ersten Satz des Zitates ausschließlich in den professionellpragmatischen Aussageanteilen und häufig in Kombination mit Vorkonstrukten (,natürlich“, ,man muss halt jetzt“) auf und erzeugt damit einerseits den Eindruck von Alternativlosigkeit, aber auch eine Entpersonalisierung der Aussage. Demgegenüber stehen die persönlichen deiktischen Partikel ,ich“ und ,wir“ in Verbindung mit der idealistischen, wertegeleiteten Subjektposition. Dieses Ringen der Subjektpositionen wird auch in ihrer Antwort auf meine Frage nach den Gründen für ihren Wechsel nach Deutschland deutlich:

„Ich habe ja irgendwann auch mal [...] Kinder bekommen, danach war das mit den Auslandsaufenthalten sowieso erstmal (.) geklärt, das geht dann ja auch nicht mehr so einfach, wenn die zur Schule gehen. [...] Die Übernahme des deutschen ${ }^{\#}$ Büros war dann ehrlich gesagt eine Karrierechance. [...] Eine Führungsposition in so einem großen Laden [...] kriegt man nicht jeden Tag angeboten und die Bedingungen sind natürlich besser, da wäre ich ja wirklich verrückt gewesen, wenn ich das nicht angenommen hätte. Das war dann mehr so eine Karriereentscheidung." (I-06: 27$)$ 
In diesem ersten Teil ihrer Antwort entsteht durch Vorkonstrukte und Nominalisierungen wie „Übernahme“ und „Karrierechance" wieder der Eindruck einer gewissen Alternativlosigkeit. Gleichzeitig schaffen diese Vorkonstrukte und Passivkonstruktionen (,war dann sowieso erstmal geklärt“, „kriegt man auch nicht jeden Tag angeboten“) eine persönliche Distanz zu ihrer „Karriereentscheidung“, die durch den Einschub ,ehrlich gesagt“ zusätzlich verstärkt wird. Während sich die Person in diesem Abschnitt durch die beschriebenen sprachlichen Mittel von ihren Aussagen distanziert, wird sie im darauffolgenden Abschnitt viel nahbarer: Die Äußerung von Gefühlen wie Freude bei der Arbeit mit Projektpartnern im Ausland und die häufigere Verwendung von persönlichen deiktischen Partikeln wie ,,ich“ und ,mir“ erzeugt eine größere Nähe zu diesen Aussageanteilen:

I: „Können Sie sich vorstellen, nochmal ins Ausland zu gehen?“"

B: „Ja, eigentlich sehr gut. [...] Weil es schon noch am meisten dem entspricht, was ich gerne mache, also wirklich die Projektpartnerarbeit ist schon noch das Schönste und ich lebe auch gerne im Ausland und das macht mir schon große Freude und ein Verwaltungsjob in Deutschland ${ }^{\#}$ ist auch nicht das Schönste im Leben (laut lachend). [...] Also ich beklage mich jetzt nicht, aber so ein schönes afrikanisches Land hat schon was." (I-06: 28-31)

Lautes Lachen als Zeichen für Ironie und der polyphone Marker ,aber“ im letzten Satz halten die pragmatisch-professionelle Subjektposition auf Distanz und rücken ihre Motivation, im Ausland direkt mit den Partnern zu arbeiten, in den Vordergrund. Zweifel und Frustrationen werden demnach zwar insgesamt durch Karriereund Familienentscheidungen ,wegrationalisiert“, aber die professionell-pragmatischen Subjektpositionen bleiben in diesem Zitat auf Abstand und werden sprachlich-formal von idealistischen Subjektanteilen überlagert. Die Rationalisierung von Karriere- und Familienentscheidungen deutet auf den Versuch hin, diese divergierenden Identitätsanteile zusammenzuführen und identitäre Fragmentierungen mit einer kohärenten Metaerzählung über das eigene Ich zu überdecken. Diese Form der Identitätsnarration mit Hilfe von Professionalisierung und Pragmatisierung ist Teil vieler Subjektivierungsprozesse und lässt sich in unterschiedlicher Ausprägung in den Erzählungen nahezu aller meiner Gesprächspartner*innen rekonstruieren.

\subsubsection{Formen der contre-conduite}

Allen bisher beschriebenen Umgangsweisen mit Sinnfragen am ersten Pol der Skala ist gemein, dass sie von wirkmächtigen Selbstführungslogiken durchzogen werden, die die Zweifel einhegen und die kohärente Erzählung über den
Sinn der eigenen Tätigkeit und damit auch über den Sinn der eigenen Identität aufrechterhalten. Demgegenüber steht am zweiten Pol der Skala ein anderer Umgang mit identitären Fragmentierungen und Zweifeln. Auch hier sind Selbstführungsmechanismen und identitäre Narrationen erkennbar, aber im Gegensatz zum ersten Pol werden Widersprüche reflektierter wahrgenommen und gegen-hegemoniale Subjektpositionen pointiert artikuliert. In diesen Fällen erfolgt häufig ein Umschwenken zu einer entwicklungskritischen Haltung:

„Man könnte ja auch sagen, man lässt das alles sein. [...] Keiner macht mehr irgendwelche Entwicklungsarbeit. Sieht dann die Welt wirklich schlechter aus? Das muss man sich ja mal überlegen. [...] Insofern, ja, klar habe ich Zweifel an der Arbeit. [...] Letztendlich muss unser Ziel ja sein, wir werden nicht mehr gebraucht. [...] (.) Jetzt werden wir aber immer noch gebraucht. Oder denken das zumindest, [...] ne, und ähm, ja, insofern, hm." (I-22: 28)

Mit Foucault können die Umgangsweisen an diesem Pol als Formen der contre-conduite (Gegen-Führung) interpretiert werden, die die Entwicklungspraxis durch ein Ausscheren aus den tradierten Denk- und Handlungsmustern im Kleinen herausfordern und anzueignen versuchen (vgl. Kap. 2).

Die Hintergrundfolie aller Facetten der Gegen-Führung bildet wie im obigen Zitat ein aus Zweifeln und identitären Fragmentierungen geborenes Unbehagen gegenüber der „Entwicklungszusammenarbeit“ oder gegenüber globalen Abhängigkeitsverhältnissen und der ,,imperialen Lebensweise" des globalen Nordens (Brand und Wissen, 2017). Während manche Personen diese Zweifel und Kritik in offensichtlich-reflektierte und teilweise kreative Formen der contre-conduite umsetzen, flackern Momente der GegenFührung bei anderen nur punktuell auf. Contre-conduite folgt also nicht immer den gleichen Mustern und Zielen, sondern äußert sich auf unterschiedliche Weise im Alltag der Entwicklungspraktiker*innen. Aus meinen Gesprächen ließ sich ein ganzes Spektrum der Gegen-Führung ableiten: Es umfasst z.B. mehr oder weniger reflektiertes Nicht-Handeln, wenn Menschen Angebote von Organisationen mit anderen Wertegrundlagen ablehnen (I-02: 22), sich weigern, für die staatliche „EZ“ zu arbeiten (I-22: 29-30), oder aus Zweifeln und Frustration dem Arbeitskontext „EZ“ insgesamt den Rücken kehren (wollen) (I-07: 27). Auch verstörende Praktiken und unangepasstes Verhalten sowie die klare Äußerung von Meinungen über Hierarchiegrenzen hinweg bilden in diesem Verständnis eine Facette von GegenFührung und Kritik, verstanden als ,die Kunst der freiwilligen Unknechtschaft, der reflektierten Unfügsamkeit“ (Foucault, 1992 [1978]: 15).

Die größte Rolle im Spektrum der Gegen-Führung spielt das Ausnutzen von Spielräumen und Möglichkeiten innerhalb der eigenen Arbeit. Charakteristisch für diese Form der 
Gegen-Führung ist, dass der Umgang mit Zweifeln und Enttäuschungen weniger über inkorporierte Logiken des Entwicklungsdiskurses erfolgt, sondern eher über Sinnzuschreibungen und Selbstführung außerhalb dieses Konstruktes. Dies wird beispielhaft an den Aussagen eines Sozialpädagogen deutlich, der einige Jahre im Kontext der „EZ“ gearbeitet hat:

„An der sozialpädagogischen Arbeit habe ich nicht gezweifelt. An der Sinnhaftigkeit von EZ habe ich insofern auch nicht richtig gezweifelt, weil ich mir diese Frage nicht stelle. Weil, wenn ich mir die wirklich (betont) stelle und ehrlich bin, dann sage ich, [...] das macht keinen Sinn. Das ist manchmal sogar wirklich kontraproduktiv. [...] Wir können uns auch gut vorstellen, nochmal ins Ausland zu gehen. [...] Und das kann ich mit einem reinen Gewissen machen, weil ich sage, okay, ich leiste da trotzdem gute Arbeit, ich mache dann sozialpädagogische Arbeit. [...] Aber nicht, weil ich glaube, dass meine Arbeit zur Entwicklung eines Landes beiträgt, das finde ich völlig lächerlich.“ (I-15: 25)

Er nutzt das Gerüst der „Entwicklungszusammenarbeit“ als Hebel, um eine anders gerahmte, ,sinnvolle“ sozialpädagogische Arbeit zu leisten. Durch das reflektierte Offenhalten von Zweifeln und Kritik in Kombination mit der pragmatischen Fokussierung auf seine konkrete Arbeit öffnet sich für ihn das Tor zur Gegen-Führung. Diese wird unter anderem in Form einer ganz praktisch-materiellen Aneignung der Entwicklungslogik während seines Arbeitsaufenthaltes mit seiner Familie in Sri Lanka ${ }^{\#}$ greifbar:

„In Sri Lanka\# hatten wir ein Haus, das echt sehr fett war. [...] Zuerst haben wir uns gefreut, und dann waren wir den ersten Tag da und am Abend begegneten wir beide uns im Haus, und guckten uns erschrocken an und meinten, wir ziehen morgen wieder aus'. Das war ganz furchtbar. Und dann haben wir überlegt, [...] dieses Haus zu einem open space [für die lokale Community, Anm. d. A.] $\mathrm{zu}$ machen [...]. Es gab einmal die Woche ein $\mathrm{Ki}-$ no bei uns [...], es war ein Veranstaltungsort, alle Leute wussten, wenn wir eine Feier haben, können wir zu denen. [...] Wir hatten immer Mitbewohner, wir hatten immer Veranstaltungen [...]. Das sind so Mechanismen, die unserem Gewissen guttun, in dem Wissen, okay, wir sind in dieser Mühle der EZ, die wir beide kritisch sehen, [...] wir ziehen viele Vorteile daraus, aber dann lass uns das zumindest kulturell sensibel machen und gucken, wie wir ein bisschen teilen können, was wir da erleben." (I-15: 65)

Diese Zitate zeigen, wie aus identitären Fragmentierungen und einem kritischen Pragmatismus das Potential für contre-conduite entsteht, d.h. im eigenen Alltag Möglichkeiten und Spielräume zu nutzen, um die wahrgenommenen Widersprüche und Zweifel in Aneignungsprozesse umzusetzen und im Kleinen Veränderungen anzustoßen. Ähnlich wie bei der Einhegung von Zweifeln und Sinnfragen am ersten Pol der Skala (Kap. 4.2.1) findet auch hier eine logische Erzählung der eigenen Identität statt; die identitäre Fragmentierung wird in diesem Fall jedoch über emanzipatorische Praktiken geflickt, die die klassische Entwicklungslogik zu transformieren versuchen. Grundlage dieses Identitätsnarrativs sind ebenso Selbstführungslogiken, die anstelle der „Entwicklungsarbeit“ die sozialpädagogische Arbeit als „gut" setzen und zum Ziel der eigenen Arbeit machen.

Bemerkenswert ist, dass das Ausnutzen von Spielräumen im Sinne der Gegen-Führung auch oder gerade durch (neoliberale) Flexibilisierungstendenzen ermöglicht wird:

„Ich habe sehr viele Freiheiten, wie ich die Arbeit gestalte. [...] Ich kann da sehr selbstbestimmt arbeiten. Ich kann mir meine Schwerpunkte raussuchen. Ich kann überlegen, ob ich in diese oder jene Richtung gehe. [...] Ich habe keine oder wenige [...] in meiner Organisation ${ }^{\#}$, die mir Steine in den Weg werfen oder mich zurückhalten oder mich sonst wie behindern." (I-09: 28)

Dieses Zitat verdeutlicht exemplarisch die doppelte Rolle von Flexibilität und eigenen Spielräumen in der Entwicklungspraxis: Sie ist sowohl ein Regierungsinstrument und damit Grundlage für die Selbstführung im Sinne der , guten Sache“ (vgl. Kap. 4.1), als auch ein Türöffner für emanzipatorische Interventionen und Gegen-Führung. In Kombination mit dem Offenhalten von Brüchen und Zweifeln ermöglicht sie nicht nur in diesen Beispielen ein partielles Heraustreten aus den üblichen Logiken der Selbstführung im Sinne des „Guten“ und einen kreativen Umgang mit (Selbst-)Zweifeln und Widersprüchen. Die Bewusstmachung dieser Spielräume bietet die Chance zur Aneignung und Herausforderung des Entwicklungsdiskurses im Kleinen und für emanzipatorische, unangepasste Interventionen im Rahmen der alltäglichen Arbeit, um so einen schleichenden Wandel anzustoßen.

Dabei ist die Grenze zwischen Selbst- und Gegen-Führung ein Graubereich. Dies wird besonders in einer weiteren Form der contre-conduite deutlich, in der es um das Ausnutzen persönlicher Einflussmöglichkeiten im Beruflichen oder im Privaten geht:

„Ich glaube, dass ich [anfangs, Anm. d. A.] idealistischer war, dass ich gedacht habe, man kann mit der Arbeit noch mehr erreichen. Und ich glaube, da bin ich inzwischen, mh, (..) ja, rationaler geworden [...]. Eigentlich ist das, was ich am meisten erreichen kann, durch mein [...] direktes Umfeld, meine Freunde, aber gerade meine Kinder, die haben natürlich notgedrungen den direkten Einfluss von mir (lachend) und den mache ich 
auch geltend (lachend). Dass die [...] sich überlegen, was sie für einen Einfluss haben durch ihr Verhalten auf [...] die Situation anderer Menschen. [...] Also es ist nicht so, dass wir nur bio kaufen und nur Textilien aus fairem Handel, und das müssten wir ja eigentlich tun, aber so konsequent sind wir nicht $[\ldots]$. Aber trotzdem ist es mir wichtig, dass meine Kinder wenigstens wissen (betont), wie es in anderen Ländern aussieht. [...] Und dass sie dafür ein Gespür entwickeln und eine Sensibilität haben.“ (I-14: 29)

Durch die Kombination aus polyphoner Satzstruktur und Vorkonstrukt wird der fließende Übergang zwischen Selbstführung und Gegen-Führung deutlich. Selbstführung findet auch hier im Sinne eines idealisierten „Guten“ statt, das „Gute" wird allerdings ähnlich wie im oberen Beispiel modifiziert: Die eingangs geäußerten Zweifel an der Wirkung von „Entwicklungszusammenarbeit“ führen einerseits zu einer gewissen Bescheidenheit in Bezug auf die zu erreichenden Ziele (vgl. auch Abb. 2). Andererseits gehen sie darüber hinaus, indem die Zweifel nicht nur hingenommen, sondern indem alternative Einflussmöglichkeiten gesucht werden. Die Folge ist eine Verschiebung des Fokus hin zu einem konsumund kapitalismuskritischen Diskurs, der Gegen-Führung im Privaten in Form von Bildung, Aufklärung und reflektierten Konsumentscheidungen ermöglicht. Gleichzeitig produziert er ebenso Selbstführungslogiken (,,und das müssten wir ja eigentlich tun"), die an das Umfeld und die eigenen Kinder weitergegeben werden. Dieses Wechselspiel aus Selbst- und Gegen-Führung füllt die Hülle des „Guten“ in beiden Beispielen nicht mit der klassischen „EZ“-Logik, sondern mit anderen machtvollen, normativen Idealen aus sozialpädagogischer Arbeit und Konsumkritik.

Logiken der Selbstführung und das Ziel der eigenen Sinnstiftung prägen demnach alle beobachteten Umgangsweisen mit Desillusionierungen und Frustrationen im Arbeitsalltag, sei es die konsequente Einhegung von Zweifeln oder der Versuch der Gegen-Führung. Ein wichtiger Unterschied besteht jedoch darin, dass die Fragmentierungen an dem ersten Pol der Skala durch identitäre Narrationen eingehegt und geflickt werden, während sie am zweiten Pol kritische Interventionen auslösen und mögliche Veränderungen anstoßen.

\section{Fazit und Ausblick}

Die wechselseitige Beziehung von Diskursen der „Entwicklung“ mit alltäglichen Arbeitserfahrungen und Erlebnissen von Entwicklungspraktiker*innen und ihre Subjektivierungsprozesse standen im Fokus dieses Beitrages. Um die komplexe (Re-)Produktion von Subjektivität durch soziale Prozesse und diskursive Praktiken sowie ihre Brüchigkeit und Kontingenz zu betonen, wurden Fragen der Identität von Entwicklungspraktiker*innen auf der Grundlage narrativbiographischer Interviews und mit gouvernementalitätstheo- retischen Ansätzen bearbeitet. Diese Vorgehensweise legt den Fokus anders als Arbeiten aus der Aidnography nicht auf die Reflexion eigener Erfahrungen oder die enge Begleitung von Personen in ihrem Arbeitsalltag. Vielmehr ergänzt sie diese ethnographischen Arbeiten durch eine Analyse der versprachlichten Reflexionen von Entwicklungspraktiker*innen über ihre alltägliche Arbeit. Im Vordergrund steht dabei das Erkennen von überindividuellen Mustern der Subjektivierung und (Selbst-)Führung, aber auch von identitären Fragmentierungen, Ambivalenzen und internen Widersprüchlichkeiten mit Hilfe von Mikromethoden der Aussagenanalyse.

Auf diese Weise wurde anhand des Interviewmaterials herausgearbeitet, dass der Entwicklungsdiskurs vermittels internalisierter Normen und Ideale des „Guten“ auf Identitätskonstruktionen einwirkt und dort über machtvolle Selbstführungsmechanismen im ,entwicklungsunternehmerischen Selbst“" (re-)produziert wird. Dabei folgt die inkorporierte Logik der „Entwicklung“ den universal gesetzten westlichhumanistischen Normen und Grundwerten. Charakteristisch für die Ausbildung von Selbstführungslogiken in der Entwicklungspraxis ist eine Kombination aus internalisierten Verantwortungsdiskursen und der normativen Aufladung und Idealisierung der eigenen Arbeit. Gleichzeitig konnte die Analyse zeigen, dass Selbstführung im Sinne des „Guten“ im Zusammentreffen mit der Entwicklungspraxis auch Zweifel, identitäre Brüche und Widersprüche hervorruft, die wiederum zu unterschiedlichen Umgangsweisen führen. Die Umgangsweisen umfassen auf der einen Seite Identitätsnarrationen, die Zweifel und Sinnfragen über Selbstführungslogiken einhegen und zerstreuen. Mit vielfältigen Schattierungen im „Dazwischen“ stehen auf der anderen Seite das Offenhalten von identitären Fragmentierungen und die pointierte Artikulation von Zweifeln, die häufig ein Umschwenken zu einer entwicklungskritischen Haltung nach sich ziehen. Diese kritische Distanz eröffnet einen Raum für widerständige Praktiken, die die Entwicklungspraxis durch ein Ausscheren aus den tradierten Denk- und Handlungsmustern im Kleinen herausfordern und anzueignen versuchen.

Hier kann Foucaults Konzept der contre-conduite den bewährten gouvernementalitätstheoretischen Rahmen in zweierlei Hinsicht sinnvoll ergänzen: Zunächst kann sie die Zwiespältigkeit und Widersprüchlichkeit im Feld der Entwicklungspraxis betonen und ein Abdriften der Analyse in eine pauschale, überhebliche Form von „Hyperkritik“ (Korf, 2018: 152) an der Entwicklungspraxis vermeiden, wie sie einigen diskurstheoretischen Arbeiten vorgeworfen wird (vgl. Korf, 2018: 155). Zudem lotet die Idee der GegenFührung die Dissonanz zwischen machtvollen, scheinbar hegemonialen Diskursen und selbstreflexiv-kritischen Entwicklungspraktiker*innen aus und zeigt damit Möglichkeiten der Transformation in einem widersprüchlichen und fragilen Feld auf. Diese vielfältigen Formen der contre-conduite im Sinne eines „So nicht regiert werden Wollens“ führen allein vielleicht nicht zur Auflösung des ,Entwicklungsdenkens", lenken aber den Blick auf Verwerfungen in Entwick- 
lungsdiskurs und -praxis und zeigen, dass diese sich nicht nur in akademisch geprägten entwicklungskritischen Debatten offenbaren, sondern in Form subtiler, subversiver Praktiken auch im „Entwicklungsalltag“. Ein stärkeres analytisches Augenmerk auf diese Mechanismen kann den Blick für Widersprüche und Widerstandspotentiale im Alltäglichen schärfen und die Möglichkeit politisch verorteter Interventionen innerhalb bestehender Machtverhältnisse aufzeigen. In Verbindung mit der in den Interviews häufig geäußerten Kritik an der imperialen Lebensweise des globalen Nordens und an (post-)kolonialen Abhängigkeitsverhältnissen eröffnen Formen der contre-conduite darüber hinaus ein emanzipatorisches Feld, das z.B. über Bildungsarbeit, die Weitergabe eigener Erfahrungen und die Sensibilisierung für globale Zusammenhänge insbesondere auch „zuhause“ seine Kraft entfalten kann, um alltägliche koloniale Denkmuster und Praktiken zu kritisieren und herauszufordern.

Datenverfügbarkeit. Die empirische Grundlage dieses Beitrages bilden narrativ-biographische Interviews, die sensible personenbezogene Daten enthalten. Da allen Interviewpartner*innen größtmögliche Anonymität und Vertraulichkeit zugesichert wurden, sind die Transkripte nicht öffentlich zugänglich. Weitere Informationen zum Interviewmaterial können bei der Autorin erfragt werden.

Interessenkonflikt. Die Autorin erklärt, dass kein Interessenkonflikt besteht.

Danksagung. Mein besonderer Dank gilt meinen Interviewpartner*innen für die wertvollen Einblicke in ihre Arbeit und ihre Beweggründe, Annika Stremmer für ihre Unterstützung bei der Transkription der Interviews sowie den Gutachter*innen für ihre konstruktiven und hilfreichen Überarbeitungshinweise.

Begutachtung. This paper was edited by Benedikt Korf and reviewed by three anonymous referees.

\section{Literatur}

Angermüller, J.: Nach dem Strukturalismus. Theoriediskurs und intellektuelles Feld in Frankreich, Transcript, Bielefeld, 2007.

Apthorpe, R.: Coda: With Alice in Aidland: A Seriously Satirical Allegory, in: Adventures in Aidland. The Anthropology of Professionals in International Development, Herausgeber: Mosse, D., Berghahn, New York, 199-219, 2011.

Ashcroft, B., Griffiths, G., und Tiffin, H. (Hrsg.): The post-colonial studies reader, Routledge, London, 2006.

Bendix, D.: Global Development and Colonial Power. German Development Policy at Home and Abroad, Rowman \& Littlefield, London, 2018.
Brand, U. und Wissen, M.: Imperiale Lebensweise. Zur Ausbeutung von Mensch und Natur im globalen Kapitalismus, Oekom, München, 2017.

Bröckling, U.: Das unternehmerische Selbst. Soziologie einer Subjektivierungsform, Suhrkamp, Frankfurt a.M., 2007.

Bröckling, U., Krasmann, S., und Lemke, T. (Hrsg.): Gouvernementalität der Gegenwart. Studien zur Ökonomisierung des Sozialen, Suhrkamp, Frankfurt a.M., 2000.

Bronner, U.: Humanitäre Helfer in Krisengebieten. Motivation, Einsatzerleben, Konsequenzen - Eine psychologische Analyse, LIT Verlag, Münster, 2003.

Bryant, R.: Non-governmental organizations and governmentality: "consuming" biodiversity and indigenous people in the Philippines, Polit. Stud.-London, 50, 268-292, 2002.

Burchell, G., Gordon, C., und Miller, P. (Hrsg.): The Foucault Effect. Studies in Governmentality, The University of Chicago Press, Chicago, 1991.

Cadman, L.: How (not) to be governed: Foucault, critique, and the political, Environ. Plann. D, 28, 539-556, 2010.

D'Aoust, A.-M.: In the Name of Love: Marriage Migration, Governmentality, and Technologies of Love, Int. Polit. Sociol., 7, 258-274, 2013.

Davidson, A. I.: In praise of counter-conduct, Hist. Hum. Sci., 24, 25-41, 2011.

Dean, M.: Governmentality. Power and rule in modern society, Sage, Los Angeles, London, 2010.

Death, C.: Counter-conducts: A Foucauldian analytics of protest, Soc. Movement Stud., 9, 235-251, 2010.

de Jong, S.: False Binaries. Altruism and Selfishness in NGO Work, in: Inside the Everyday Lives of Development Workers: The Challenges and Futures of Aidland, Herausgeber: Fechter, A.-M. und Hindman, H., Kumarian Press, Sterling, 21-40, 2011.

Ducrot, O.: Le dire et le dit, Les éditions de Minuit, Paris, 1984.

Dzudzek, I.: Kreativpolitik. Über die Machteffekte einer neuen Regierungsform des Städtischen, Transcript, Bielefeld, 2016.

Eriksson Baaz, M.: The paternalism of partnership. A postcolonial reading of identity in development aid, Zed Books, London, 2005.

Escobar, A.: Encountering development. The making and unmaking of the Third World, Princeton University Press, Princeton, 1995.

Fechter, A.-M. und Hindman, H. (Hrsg.): Inside the Everyday Lives of Development Workers: The Challenges and Futures of Aidland, Kumarian Press, Sterling, 2011.

Ferguson, J. und Gupta, A.: Spatializing states: toward an ethnography of neoliberal governmentality, Am. Ethnol., 29, 981-1002, 2002.

Foucault, M.: Der Wille zum Wissen. Sexualität und Wahrheit I, Suhrkamp, Frankfurt a.M., 1983 [1976].

Foucault, M.: Das Subjekt und die Macht, in: Michel Foucault. Jenseits von Strukturalismus und Hermeneutik, Herausgeber: Dreyfus, H. L. und Rabinow, P., Beltz Athenäum, Frankfurt a.M., 243-261, 1987 [1983].

Foucault, M.: Was ist Aufklärung?, in: Ethos der Moderne, Foucaults Kritik der Aufklärung, Herausgeber: Erdmann, E., Forst, R., und Honneth, A., Campus, Frankfurt a.M., 35-54, 1990.

Foucault, M.: Was ist Kritik?, Merve Verlag, Berlin, 1992 [1978]

Foucault, M.: Sicherheit, Territorium, Bevölkerung. Geschichte der Gouvernementalität I. Vorlesung am Collège de France 1977_ 
1978, Herausgeber: Sennelart, M., Suhrkamp, Frankfurt a.M., 2006a [1978].

Foucault, M.: Die Geburt der Biopolitik. Geschichte der Gouvernementalität II. Vorlesung am Collège de France 1978-1979, Herausgeber: Sennelart, M., Suhrkamp, Frankfurt a.M., 2006b [1979].

Füller, H. und Marquardt, N.: Sicherstellung von Urbanität. Innerstädtische Restrukturierung und soziale Kontrolle in Downtown Los Angeles, Westfälisches Dampfboot, Münster, 2010.

Gibson-Graham, J. K.: The End of Capitalism (As We Knew It). A Feminist Critique of Political Economy, University of Minnesota Press, Minneapolis, 2006a [1996].

Gibson-Graham, J. K.: A Postcapitalist Politics, University of Minnesota Press, Minneapolis, 2006b.

Glasze, G.: Vorschläge zur Operationalisierung der Diskurstheorie von Laclau und Mouffe in einer Triangulation von lexikometrischen und interpretativen Methoden, Forum Qualitative Sozialforschung, 8, online aufrufbar: http: //www.qualitative-research.net/index.php/fqs/article/view/239 (letzter Zugriff: 16 July 2011), 2007.

Gould, J.: Introducing Aidnography, in: Ethnographies of Aid. Exploring development texts and encounters, Herausgeber: Gould, J. und Marcussen, H. S., Roskilde University, Roskilde, 1-14, 2004.

Harrison, E.: Beyond the looking glass? "Aidland" reconsidered, Crit. Anthropol., 33, 263-279, 2013.

Hart, G.: Geography and development: critical ethnographies, Prog. Hum. Geog., 28, 91-100, 2004.

Hindman, H.: The Hollowing Out of Aidland: Subcontracting and the New Development Family in Nepal, in: Inside the Everyday Lives of Development Workers: The Challenges and Futures of Aidland, Herausgeber: Fechter, A.-M. und Hindman, H., Kumarian Press, Sterling, 169-191, 2011.

Kapoor, I.: The Postcolonial Politics of Development, Routledge, London, 2008.

Kiesel, T. und Bendix, D.: White Charity: Eine postkoloniale, rassismuskritische Analyse der entwicklungspolitischen Plakatwerbung in Deutschland, Peripherie, 120, 482-95, 2010.

Korf, B.: Die Ordnung der Entwicklung: Zur Ethnographie der Entwicklungspraxis und ihrer ethischen Implikationen, Geogr. Z., 92, 208-226, 2004.

Korf, B.: Die Ironie der Entwicklungstheorie, Geogr. Z., 106, 150174, 2018.

Lemke, T.: Gouvernementalität und Biopolitik, Springer VS, Wiesbaden, 2007.

Li, T. M.: The Will to Improve. Governmentality, Development, and the Practice of Politics, Duke University Press, Durham, London, 2007.

Linnemann, K.: Die Gouvernementalität widerständiger Alltagspraktiken: Eine konzeptionelle Annäherung an Postwachstum, Subjektivierung und alltägliches Gegen-Führen, Z. Wirtschaftsgeogr., 62, 233-245, https://doi.org/10.1515/zfw-20170009, 2018.

Linnemann, K. und Reuber, P.: Der lange Schatten der Moderne: Entwicklungs- und geopolitische Diskurse deutscher Hilfsorganisationen, Geogr. Z., 103, 1-18, 2015.

Lossau, J.: Die Politik der Verortung. Eine postkoloniale Reise zu einer anderen Geographie der Welt, Transcript, Bielefeld, 2002.
Marquardt, N.: Räume der Fürsorge. Regieren der Wohnungslosigkeit im betreuten Wohnen, Geogr. Z., 101, 148-165, 2013.

Mattissek, A.: Die neoliberale Stadt. Diskursive Repräsentationen im Stadtmarketing deutscher Großstädte, Transcript, Bielefeld, 2008.

Mattissek, A.: Die Aussagenanalyse als Mikromethode der Diskursforschung, in: Handbuch Diskurs und Raum. Theorien und Methoden für die Humangeographie sowie für die sozial- und kulturwissenschaftliche Raumforschung, Herausgeber: Glasze, G. und Mattissek, A., Transcript, Bielefeld, 279-291, 2009.

McDonald, J.: The neoliberal project and governmentality in rural Mexico: emergent farmer organization in the Michoacan Highlands, Hum. Organ., 58, 274-84, 1999.

McEwan, C.: Postcolonialism and development, Routledge, London, 2009.

Mezzadra, S., Reid, J., und Samaddar, R. (Hrsg.): The Biopolitics of Development. Reading Michel Foucault in the Postcolonial Present, Springer, New Delhi, 2013.

Mignolo, W. D.: Epistemischer Ungehorsam. Rhetorik der Moderne, Logik der Kolonialität und Grammatik der Dekolonialität, Turia + Kant, Wien, 2012 [2006].

Mignolo, W. D. und Walsh, C. E.: On Decoloniality: Concepts, Analytics, Praxis, Duke University Press, Durham, London, 2018.

Mosse, D. (Hrsg.): Adventures in Aidland. The Anthropology of Professionals in International Development, Berghahn, New York, Oxford, 2011a.

Mosse, D.: Introduction: The Anthropology of Expertise and Professionals in International Development, in: Adventures in Aidland. The Anthropology of Professionals in International Development, Herausgeber: Mosse, D., Berghahn, New York, Oxford, 1-31, $2011 b$.

Neuburger, M. and Schmitt, T.: Editorial Theorie der Entwicklung - Entwicklung der Theorie Post-Development und Postkoloniale Theorien als Herausforderung für eine Geographische Entwicklungsforschung, Geogr. Helv., 67, 121-124, https://doi.org/10.5194/gh-67-121-2012, 2012.

Odysseos, L., Death, C., und Malmvig, H. (Hrsg.): CounterConduct in Global Politics: Theorising the Subjects and Practices of Contesting Conduct, Global Society, 30, 2016.

Opitz, S.: Gouvernementalität im Postfordismus. Macht, Wissen und Techniken des Selbst im Feld unternehmerischer Rationalität, Argument-Verlag, Hamburg, 2004.

Pêcheux, M.: Language, semantics and ideology. Stating the obvious, Palgrave Macmillan, London, Basingstoke, 1983 [1975].

Polman, L.: Die Mitleidsindustrie. Hinter den Kulissen internationaler Hilfsorganisationen, Campus-Verlag, Frankfurt a.M., 2010.

Przyborski, A. und Wohlrab-Sahr, M.: Qualitative Sozialforschung. Ein Arbeitsbuch, Oldenbourg Wissenschaftsverlag, Berlin, Boston, 2014

Pütz, R. und Rodatz, M.: Kommunale Integrations- und Vielfaltskonzepte im Neoliberalismus. Zur strategischen Steuerung von Integration in deutschen Großstädten, Geogr. Z., 101, 166-183, 2013.

Quijano, A.: Coloniality of Power and Eurocentrism in Latin America, Int. Sociol., 15, 215-232, 2000.

Raco, M. und Imrie, R.: Governmentality and Rights and Responsibilities in Urban Policy, Environ. Plann. A, 32, 2187-2204, 2000 
Rosol, M.: On resistance in the post-political city: conduct and counter-conduct in Vancouver, Space and Polity, 18, 70-84, 2014.

Rosol, M.: Governing Cities through Participation: A Foucauldian analysis of CityPlan Vancouver, Urban Geogr., 36, 256-276, 2015.

Rossi, B.: Revisiting Foucauldian Approaches: Power Dynamics in Development Projects, J. Dev. Stud., 40, 1-29, 2004.

Roth, S.: The Paradoxes of Aid Work. Passionate professionals, Routledge, Abingdon, New York, 2015.

Ruwanpura, K. N. und Hollenbach, P.: From compassion to the will to improve: Elision of scripts? Philanthropy in post-tsunami Sri Lanka, Geoforum, 51, 243-251, 2014.

Schipper, S.: Genealogie und Gegenwart der ,unternehmerischen Stadt“. Neoliberales Regieren in Frankfurt am Main zwischen 1960 und 2010, Westfälisches Dampfboot, Münster, 2013.

Sharp, J. P.: Geographies of Postcolonialism. Spaces of Power and Representation, Sage, London, 2009.
Shutt, C.: Power in Aid Relationships: A Personal View, IDS Bulletin, 37, 79-87, 2006.

Sidaway, J. D.: Spaces of postdevelopment, Prog. Hum. Geog., 31, 345-361, 2007.

Stirrat, R. L.: Mercenaries, Missionaries and Misfits. Representations of Development Personnel, Crit. Anthropol., 28, 406-425, 2008.

Strüver, A.: The Production of Geopolitical Regionalizations and Gendered Images through Global Aid Organizations, Geopolitics, 12, 680-703, 2007.

Tazzioli, M.: Spaces of Governmentality. Autonomous Migration and the Arab Uprisings, Rowman \& Littlefield, London, 2014.

Walters, W.: Governmentality. Critical encounters, Routledge, Abingdon, New York, 2012.

Ziai, A.: Development Discourse and Global History. From Colonialism to the Sustainable Development Goals, Routledge, London, New York, 2016. 